\title{
Particle Generation during Photoresist Dissolution
}

\author{
Siddharth Chauhan ${ }^{a}$, Mark Somervell ${ }^{\mathrm{b}}$, Michael Carcasi ${ }^{\mathrm{b}}$, Steven Scheer ${ }^{\mathrm{b}}$, \\ Roger T. Bonnecaze ${ }^{a}$, Chris Mack $^{c}$ and C. Grant Willson ${ }^{a}$ \\ ${ }^{a}$ Department of Chemical Engineering, The University of Texas at Austin, Austin, TX 78712, USA; \\ ${ }^{\mathrm{b}}$ Tokyo Electron America LTD, 2400 Grove Blvd, Austin, Texas 78741 USA; \\ ${ }^{c}$ Lithoguru.com, 1605 Watchhill Rd., Austin, TX 78703, USA
}

\begin{abstract}
A lattice-type Monte Carlo based mesoscale model and simulation of the lithography process has been described previously [1]. The model includes the spin coating, post apply bake, exposure, post exposure bake and development steps. This simulation has been adapted to study the insoluble particle generation that arises from statistically improbable events. These events occur when there is a connected pathway of soluble material that envelops a volume of insoluble material due to fluctuations in the deprotection profile that occur during the post exposure bake [2]. Development erodes the insoluble material into the developer stream as an insoluble particle. This process may produce a cavity on the line edge that can be far larger than a single polymer molecule. The insoluble particles generated may coalesce in developer to form large aggregates of insoluble material that ultimately deposit on the wafer surface and the tooling. The recent modifications made in mesoscale models for the PEB and dissolution steps, which have enabled this study are briefly described. An algorithm that was used for particle detection in the current study is also discussed. The effect of the resist formulation and the different lithographic steps, namely, exposure, post exposure bake and development, on the extent of particle generation is analyzed. These simulations can be used to set process variables to minimize the extent of particle generation.
\end{abstract}

Keywords: Mesoscale modeling, particle generation

\section{INTRODUCTION}

Defectivity has always been a key concern for the semiconductor industry. Because defects lead to lost yield and therefore lost revenue, defects are being investigated in every manufacturing facility in the world. Although lithography processes are relatively clean, they are not perfect and all have some level of defectivity. For example, recent focus on immersion lithography has resulted in a large number of new studies directed at understanding the new sources of defectivity that are introduced by the immersion process. An important class of lithographic defects is "post-develop defects", that are generated during development of the photoresist. It has been proposed that these insoluble residues are generated by an erosion event that occurs when there is generated by chance, a connected pathway of soluble material that envelops or surrounds an insoluble volume [2]. Such residues or particles are inherently insoluble photoresist material that would have a tendency to redeposit on the wafer or the tools. Any drop in the $\mathrm{pH}$ of the solution, like that which occurs during the rinse step, would aggravate the problem by further reducing the solubility of the particles already suspended in the solution. These statistical excursions of the development path during photoresist dissolution can produce a cavity on the line edge that is far larger than the volume of a single polymer molecule and hence can produce low frequency, high amplitude line edge roughness (LER).

Simulation studies have proven to be a time and cost effective approach to solving several engineering problems. Lithographic simulation and Monte Carlo techniques are now being used throughout the industry to predict CD error budgets, process windows, and other important lithographic parameters, but the subject of defectivity has largely remained untouched by these simulations. Since defectivity is a stochastic process, and Monte Carlo simulations inherently make use of stochastics, there should be some way to link the two together in order to study the fundamental nature of defect formation in the lithographic process. This paper describes an attempt at making that link.

Advances in Resist Materials and Processing Technology XXVII, edited by Robert D. Allen, Mark H. Somervell, Proc. of SPIE Vol. 7639, 763933 - (c) 2010 SPIE · CCC code: 0277-786X/10/\$18 · doi: 10.1117/12.848424 
A comprehensive, 3D photoresist-dissolution model is essential for capturing the excursions from the developer pathway during the dissolution process and their dependency on various process variables. A mesoscale, dynamic Monte Carlo model for photoresist dissolution based on the molecular level interactions between distinct components of the resist and the developer has been developed $[3,4]$. The stochastic variations in the deprotection profile produced during the post exposure bake (PEB) that are captured by this model are critical for this study. The reactive transport processes that occur during PEB have been simulated based on a new proposed transient mechanism for acid diffusion [5]. These improvements in the simulator have enabled detection of the volumes of insoluble material eroded into the developer near the line edge during development. These improvements may also provide access to valuable information about the relative contributions of different lithographic steps to LER and thereby insight into the fundamental causes of LER.

\section{MESOSCALE MODELING}

The present simulation work relies on mesoscale modeling, which is based on molecular scale interactions and inherently provides a stochastic picture of the imaging process. The model photoresist studied consists of copolymers of $p$ hydroxystyrene and $t$-butyloxycarbonyloxystyrene. A three-dimensional cubic lattice structure was created to simulate the photoresist film. Each cell in the lattice is assigned either as a monomer (where a string of connected monomer cells represents a polymer chain), PAG, quencher or a void. A completely random configuration is obtained using a Monte Carlo approach with periodicity in the given direction. Next, the most relevant and recently revised simulation modules, namely PEB and dissolution are briefly described.

\subsection{Post Exposure Bake}

Acid diffusion in a chemically amplified resist amplifies the variation in average protection level near the interface of exposed-unexposed region. The roughness in the protection level is believed to be a major contributor to the line edge roughness observed after the dissolution step. Needless to say, an accurate acid diffusion model is therefore critical for any LER study. Experimental results have demonstrated a fast acid diffusion for the first $\sim 1$ min of the PEB followed by a tremendous slow down in subsequent acid diffusion rate [6]. The reported diffusivity of acid in pure poly(phydroxystyrene) is very small $\left(<10^{-7} \mathrm{~cm}^{2} / \mathrm{s}\right)$ at typical PEB temperature and would result in a mean diffusion length of only $\sim 1 \mathrm{~nm}$. Postnikov et al. proposed a Reaction Front Propagation model to explain the observed acid diffusion behavior [7]. They postulated that the deprotection reaction causes local, transient enhancement of acid diffusivity, which results in high acid diffusion at the reaction front but this enhanced diffusivity lasts only for a very short period of time.

The concept of reaction enhanced diffusivity could be explained for APEX type $248 \mathrm{~nm}$ resists by invoking transient free volume generation during the deprotection reaction as the mechanism for diffusivity enhancement [1]. Clearly the loss of volatile products such as isobutylene that occurs during PEB could cause a local and transient plasticization of the film and/or could generate a transient and local volume of reduced density. Of course this low density condition is short lived because the film collapses to produce the familiar relief image that is observed after the PEB. Unfortunately, this mechanism for the transient enhancement of diffusivity does not apply to methyladamantyl protected $193 \mathrm{~nm}$ resists where the by-product of the deprotection reaction is not volatile as it is in APEX type $248 \mathrm{~nm}$ resists. Recently, we have explored a different explanation for this phenomenon that relies on the heat from the exothermic deprotection reaction elevating the local temperature above the glass transition temperature $\left(\mathrm{T}_{\mathrm{g}}\right)$ of the polymer and thus enhancing the acid diffusivity in a local region $[5,8]$. The diffusivity or permeance of a polymer film can change by several orders of magnitude when the local temperature exceeds $T_{\mathrm{g}}$. As time progresses the heat generated by the reaction diffuses away from the local region until the local temperature drops below $T_{g}$, thus rendering only a transient acid diffusivity enhancement. Any acid molecule that happens to be in a volume of polymer above $T_{g}$ is able to diffuse rapidly. During this diffusion, if an acid molecule encounters any protected site it can catalyze another deprotection reaction and it then continues to diffuse. However if the reaction front has moved forward and there are no reaction sites available for a given acid molecule, then the matrix will cool below $\mathrm{T}_{\mathrm{g}}$ and that acid molecule will diffuse no further. Eventually, all the acid molecules become trapped and the reaction front stops, not because of loss of chemical reactivity, but because the acid catalyst is immobilized. 
The temperature profile around the deprotection site was estimated by solving the heat equation in spherical coordinates with an isolated point heat source [9]. The local diffusivity can be estimated on the basis of the empirical relationship between the temperature and the diffusivity [6]. However for simplicity, we have used a binary profile for acid diffusion i.e. acid is allowed to diffuse only when the local temperature is above the given temperature and below that temperature it is immobile. In order to facilitate the computation, the present model allows an acid molecule to diffuse through and share a lattice site with other resist components. Thus, the excluded volume condition is relaxed, a characteristic which is inherently assumed in continuum models.

\subsection{Photoresist Dissolution}

At the onset of the development step, a developer film is added to the lattice structure with lattice grids extended into the developer film. The dissolution model assumes that base is readily available for deprotonation at polymer acidic sites and there is no depletion zone. When the aqueous base (developer) comes in contact with the polymer, deprotonation of the ionizable acidic sites causes accumulation of a negative charge at the polymer-developer interface [10]. The charged surface results in the depletion of hydroxide ions near the interface, and an electric double layer is formed. The PoissonBoltzmann equation and the equilibrium conditions are used to yield the ion concentration at the charged surface and the corresponding fractional surface ionization. Thereafter the surface is fractionally ionized and the dynamic equilibrium is maintained by considering ionization/deionization steps at the polymer-developer interface. Polymer chain motion and chain solvation at the film-developer interface are simulated using the Metropolis algorithm where the probability of a move is based on the energetics of interactions between different species in the polymer and the developer. Mesoscale modeling using this approach not only accounts for the favorable interaction between the ionized part of the polymer and the developer solution to render the polymer soluble as in the Critical Ionization model, but also takes into account the chain configuration and the interactions of the non-ionized part of the chain with the developer and the polymer film. The interaction parameters for different species in the system were based on experimentally determined interfacial energy values and the ionized polymer-developer interaction parameter was determined by calibrating the simulated dissolution rate response to polymer molecular weight and developer concentration against measured values $[3,11,12,13]$.

\section{INSOLUBLE PARTICLE GENERATION}
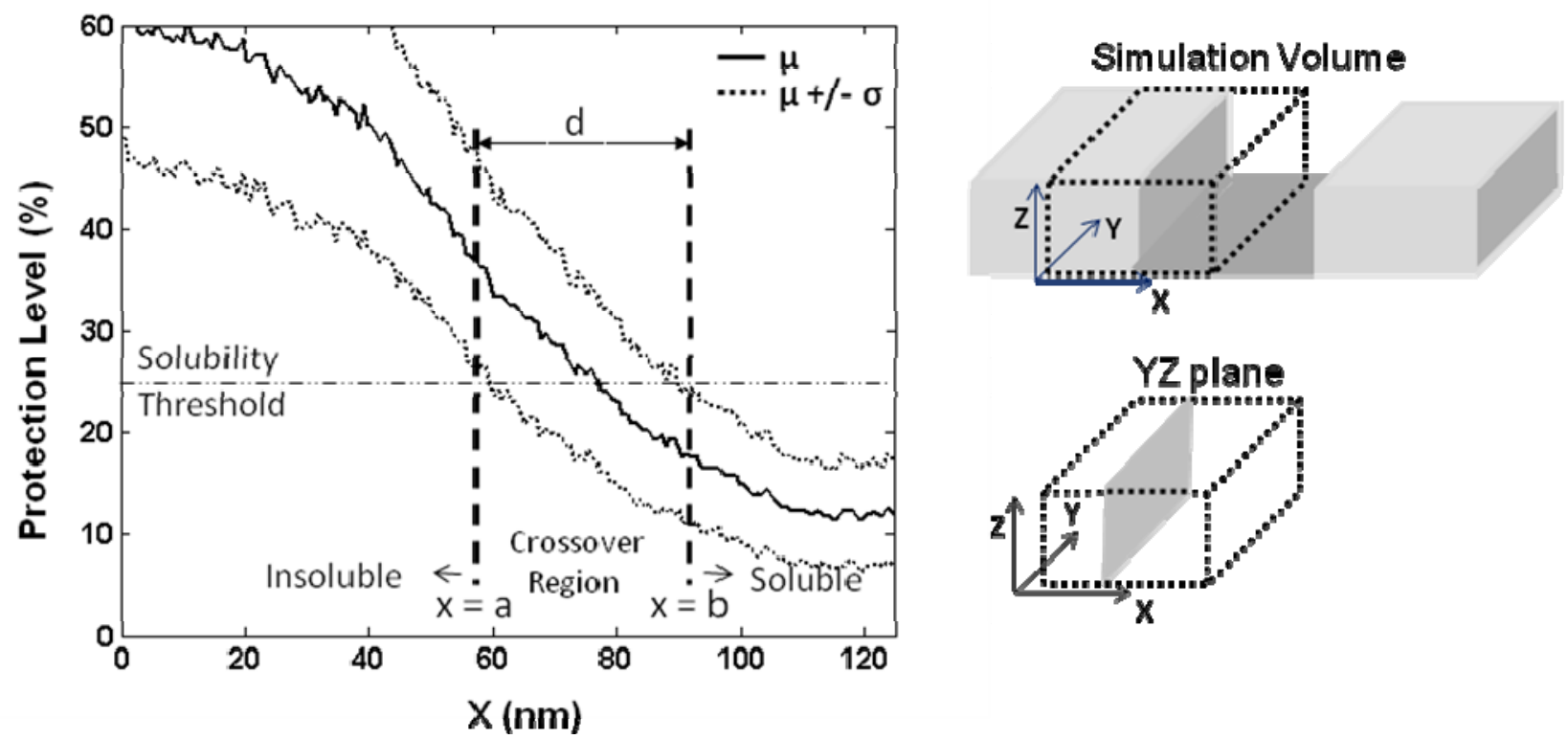

Figure 1. The average protection level is shown with a solid line (averaged over $\mathrm{y}-\mathrm{z}$ plane at any given $\mathrm{x}$ ). The dotted lines show the limits of one standard deviation in the protection level at different $\mathrm{y}$ along the $\mathrm{y}-\mathrm{z}$ plane as a function of the $\mathrm{x}$ dimension. 
An aerial image profile produces an acid gradient in the exposed resist film. During the post exposure bake (PEB), the acid gradient results in a corresponding gradient in the average protection level (chemical gradient). There is a certain degree of stochastic variation in the average blocking level. Figure 1 shows the average protection level (averaged over $\mathrm{y}-\mathrm{z}$ plane at any given $\mathrm{x}$ ) with $+/-\sigma$ variation in the protection level at different $\mathrm{y}$ along the $\mathrm{y}-\mathrm{z}$ plane as a function of ' $\mathrm{x}$ ' dimension of a feature. For the sake of argument, assume that the polymer solubility switch occurs at $25 \%$ protection level. The protection level to the left of $\mathrm{x}=\mathrm{a}$ is higher than the solubility threshold and therefore the resist volume is insoluble. On the other hand, the protection level to the right of $x=b$ is lower than the solubility threshold, implying that everything to the right of $\mathrm{x}=\mathrm{b}$ is soluble. However, there exists a solubility crossover region with a finite width ' $\mathrm{d}$ ' between $\mathrm{x}=\mathrm{a}$ and $\mathrm{x}=\mathrm{b}$ where part of the resist volume is soluble (protection level lower than threshold limit) while the rest of it is inherently insoluble (protection level above the threshold limit). Solvation of each polymer chain within the soluble volume paves a path that allows the developer to penetrate and interact with the lower chain. If there exists a connected pathway of soluble material that envelopes a volume of insoluble material in the solubility crossover region, development erodes the insoluble material into the developer stream as an insoluble particle. It is worthy to note that this analysis is a simplistic representation of the current problem. Here variation in protection level in ' $z$ ' direction has not been considered. That is, the influence of film absorbance has been neglected. In reality, the possibility of connected pathways and the statistics (size distribution, protection level, etc.) of insoluble particles generated depends on the actual 3D configuration of resist components and the development mechanism. Therefore 3D mesoscale models for PEB and dissolution steps discussed in earlier sections are crucial in this study.

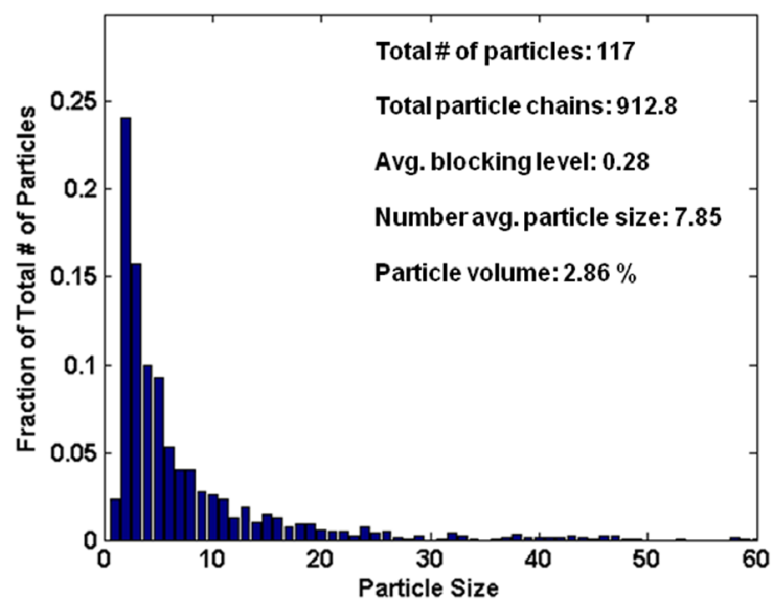

(a)

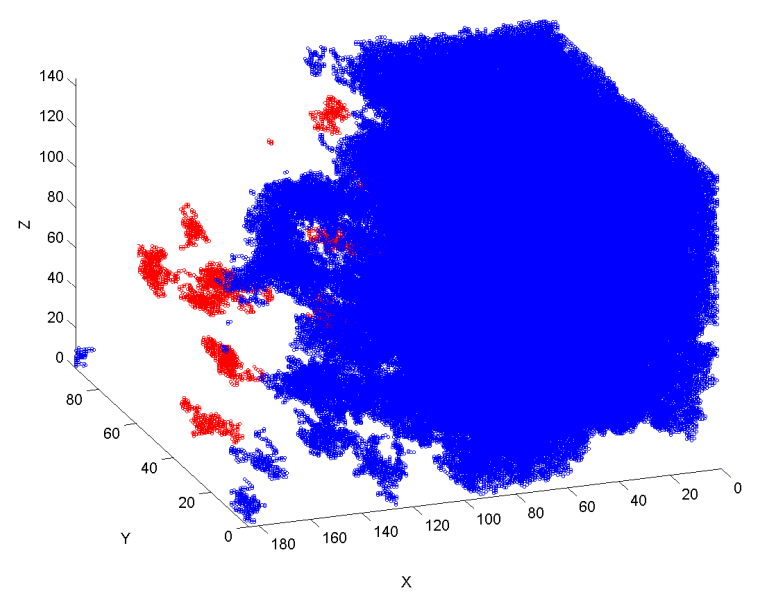

(b)

Figure 2. (a) Particle size distribution for a typical simulation case (average of 10 runs), where the number of polymer chains correspond to the particle size. Also shown is the post analysis data that can be obtained after the simulation run. (b) A snapshot of one of the simulation run showing the particles generated during the photoresist dissolution.

The detection of the particles during dissolution run is also critical and requires a suitable search algorithm. The network of chains in contact with the substrate defines the bulk resist film and any group of chains detached from the resist film but not dissolved yet is considered as a particle. The connectivity between the two chains is determined by a minimum of one direct contact between the chains or indirect contact through a series of chains. A simplistic but computationally expensive approach for particle detection would then be to scan all the chains for connectivity using any standard search algorithm such as the breadth first search algorithm. Actually, the connectivity of only the interfacial chains, i.e. those outer chains that are in direct contact with developer, is the sufficient and necessary condition for no particle generation. The interfacial chains are a small fraction of the total number of chains and therefore considering them only cuts down the search time considerably. The interfacial chains are tracked and updated at each Monte Carlo step and are periodically scanned for their connectivity through interfacial chains. If all interfacial chains are connected then there exists no particle and the particle search ends. When the interfacial chains are not all connected, then all the chains (interfacial as well as bulk chains) must be scanned for their connectivity to identify the particle. This step is computationally expensive but rare and thus the costly redundancy of scanning all the chains each time is avoided. Finally, particle details are recorded and the particle is removed from the lattice structure for the rest of the simulation. 
Once a particle has been generated, it could settle on the tooling, on the wafer, be rinsed away during the rinsing process. Investigating the fate of the particles requires the longer tracking and is beyond the scope of the current work. Figure 2 shows the size-distribution of the particles generated during a photoresist dissolution run based on the typical simulation inputs outlined in the next section. Here particle "size" is actually the number of polymer chains in the particle. As expected, there is a large number of small particles and fewer big particles. To give some prospective to the volume, a single chain occupies $\sim 27 \mathrm{~nm}^{3}$ in the simulation lattice structure. Once a simulation run is over, different statistics can be employed to do a post-analysis of the particle generation study. Shown in Figure 2 are the total number of particles, average blocking of the particles, the number average size of the particles and volume \% of particles generated. Also shown is a snapshot of the dissolution run showing the particles generated. For the purposes of this paper, the metric used to quantify particle generation is the volume percent of the insoluble particles in the total resist volume dissolved.

\section{RESULTS AND DISCUSSIONS}

A film of APEX type resist with dimensions of $130 \mathrm{~nm}$ x $250 \mathrm{~nm}$ x $100 \mathrm{~nm}$ (half pitch x line length x film thickness) was simulated using a $184 \times 356$ × 144 lattice structure with periodic boundary conditions only along the line length. The following resist formulation and process conditions, unless otherwise specified, were used in the particle generation study. The resist that was simulated is formulated from a polymer with degree of polymerization of $80,60 \%$ protection level, pKa of 10.25, $5 \mathrm{wt} \%$ PAG loading and 20\% quencher loading (relative to PAG moles). The exposure conditions involve NILS equals to 2.41, dose to size with Dill C parameter as $0.03 \mathrm{~cm}^{2} / \mathrm{mJ}$ and the image in resist using PROLITH for typical $\mathrm{KrF}$ resist. The PEB time and the temperature were $60 \mathrm{~s}$ and $90^{\circ} \mathrm{C}$ respectively. A development time of $30 \mathrm{~s}$ with standard $0.26 \mathrm{~N}$ developer concentration was used in the development step. Typical results reported are an average of 10 simulation runs. Next, the parametric study is presented for polymer formulation (degree of polymerization, protection level), exposure (dose, NILS), PEB (PAG/ Quencher loading, PEB time) and development (developer concentration, polymer hydrophilicity).

\subsection{Polymer Formulation}

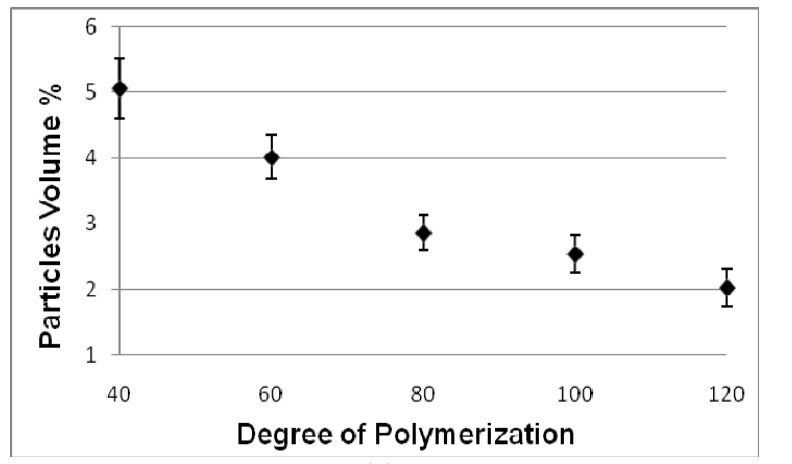

(a)

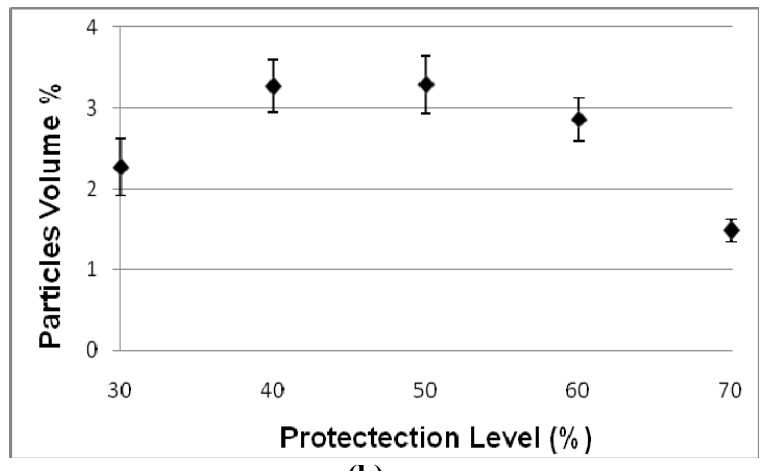

(b)

Figure 3. Particle volume $\%$ as a function of (a) degree of polymerization; and (b) protection level of the polymer.

Figures 3(a) and 3(b) quantify the particle generation for a resist polymer as a function of degree of polymerization and composition. The results indicate that the volume percentage of insoluble particles decreases with increasing degree of polymerization. The higher degree of polymerization enhances the extent of chain entanglement in the polymer matrix and the increased entanglement makes the erosion phenomenon less probable, thereby producing fewer insoluble particles. Figure 3(b) shows that the particle generation first increases and then decreases with the protection level of the polymer. The farther the polymer composition is from the solubility switch protection level, the higher the possibility is of finding an insoluble volume in the solubility crossover region. The solubility switch for APEX resists occurs below 30\% protection level under normal development conditions, so there is an increase in particle generation as protection level initially increases above $30 \%$. However, the particle generation process depends not only on the existence of an insoluble volume but also on the existence of a connected pathway of soluble material surrounding 
the particle to "erode" the particle from the bulk. The further increase in the protection level reduces the developer penetration significantly and thus suppresses particle generation. Therefore there exists a range of protection levels where the particle generation is at a maximum. Ideally, the resist should be formulated with a polymer protection level outside of that range depending on other process constraints.

\subsection{Exposure}

Figures 4(a) and 4(b) show the particle formation as a function of the normalized image log slope (NILS) of the aerial image and the exposure dose. An aerial image with a higher NILS produces a steeper chemical gradient, narrowing the solubility switch crossover region, which results in a lower fraction of insoluble material. The higher dose increases the acid concentration and a similar argument holds as for the effect of higher PAG loading discussed in the subsequent section. It is worthy to note that in this case exposure dose is varied from the dose to size and therefore the feature dimension would vary.

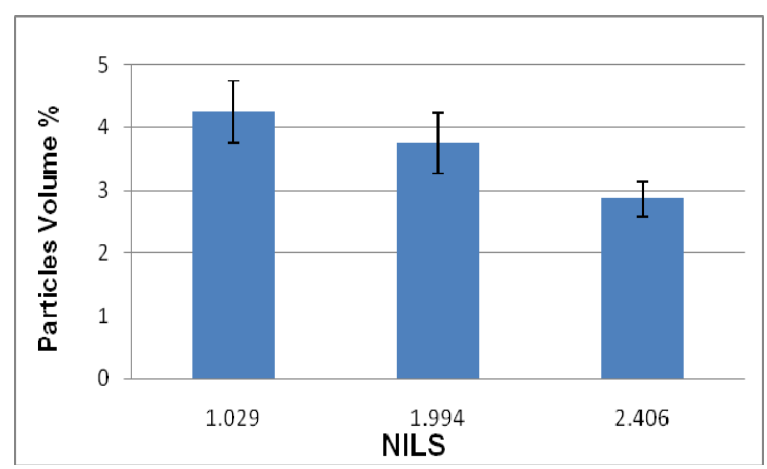

(a)

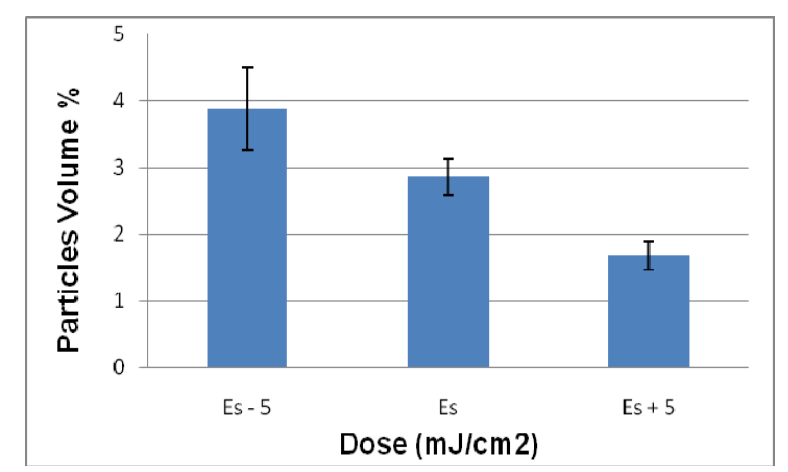

(b)

Figure 4. Particle volume $\%$ as a function of (a) normalized image log slope (NILS); and (b) exposure dose. Es represents the exposure dose to size.

\subsection{Post Exposure Bake}

In Figures 5(a) and 5(b) the percent of particle volume is plotted against PAG and quencher loading. It can be seen that higher PAG and quencher loading minimize the fractional volume of insoluble particles. The higher PAG loading yields higher acid concentration, which improves the chemical gradient and thus reduces the width of the crossover region between completely soluble and insoluble region (as illustrated in Figure 6). Likewise adding quencher (base) to the system effectively suppresses the fluctuations in the protection profile near the feature edge by reducing the concentration of acid molecules in that region. Figure 7 shows the particle volume \% for different PEB times. The volume percent of particles first decreases and then increases with an increase in PEB time. This trend suggests that there may be an optimum PEB time that would minimize particle formation.

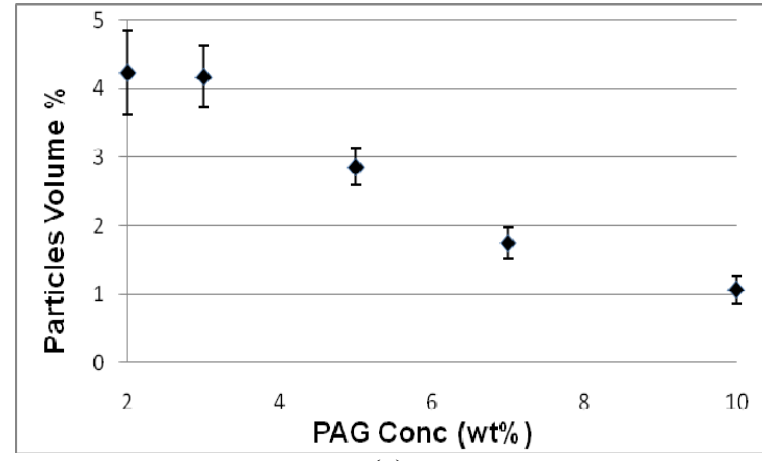

(a)

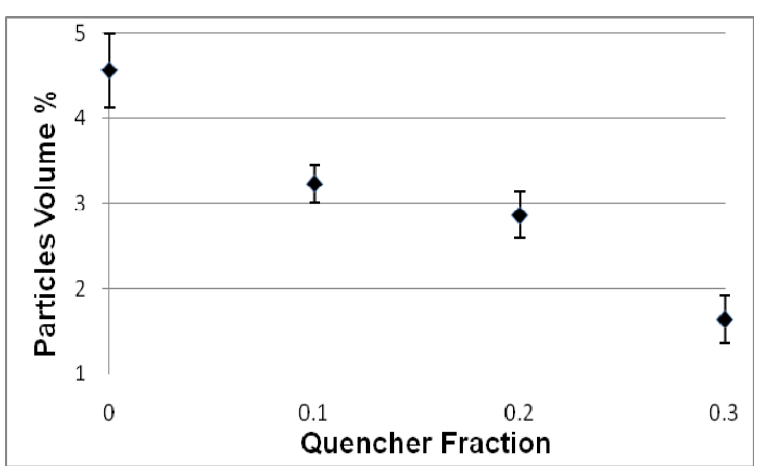

(b)

Figure 5. Particle volume $\%$ as a function of PAG concentration (wt $\%$ to the polymer) and quencher loading (fraction of total PAG moles) 


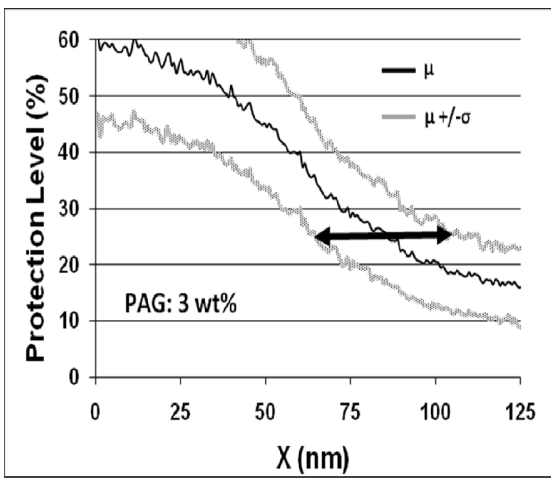

(a)

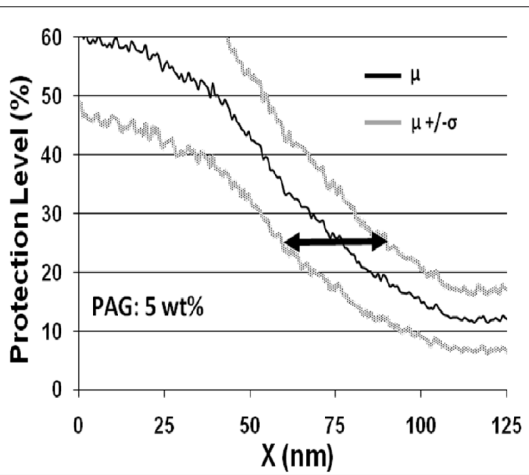

(b)

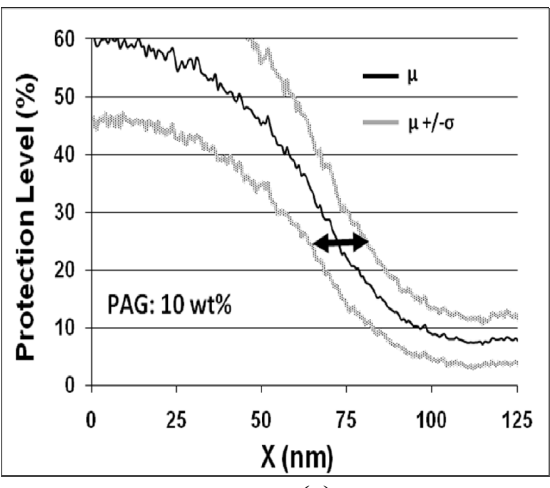

(c)

Figure 6. The chemical gradient in the film for different PAG concentration (a) $3 \mathrm{wt} \%$, (b) $5 \mathrm{wt} \%$, and (c) $10 \mathrm{wt} \%$ illustrating the change in width of the solubility crossover region. The three curves in each figure represent the mean protection level and the limits of one standard deviation in each direction.

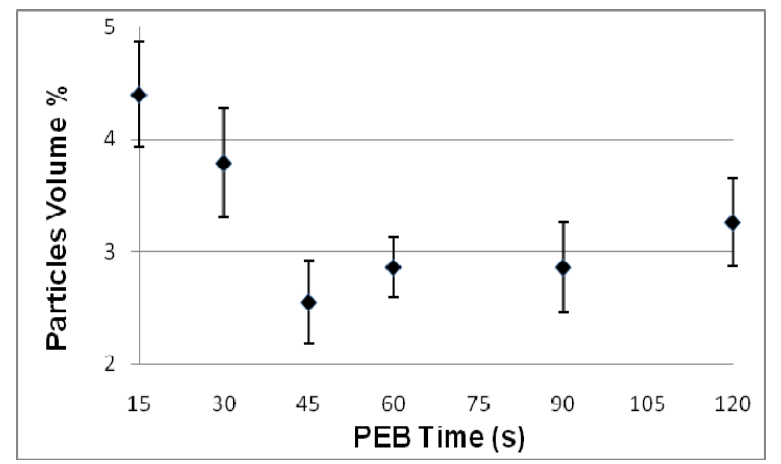

Figure 7. Particle volume $\%$ as a function of post exposure bake time

\subsection{Dissolution}

The exposure and PEB process conditions directly affect the chemical gradient and consequently the particle generation phenomenon. Given the chemical gradient profile in the exposed film, the dissolution process can be altered to minimize particle generation during the photoresist dissolution. Figures $8(\mathrm{a})$ and $8(\mathrm{~b})$ illustrate the effect of parameters related to the important photoresist dissolution step on particle generation. Increasing the developer concentration and the increment in hydrophilicity of polymer components (unprotected/protected part of the polymer chain) serve to suppress particle generation. The higher developer concentration increases a favorable interaction between the developer and the ionized part of the polymer chain. Hence, the more concentrated developer is able to solvate chains/particles with higher protection levels, which would have been insoluble otherwise. This also means that the water rinse step is a source of particle generation due to the sudden reduction in $\mathrm{pH}$ of the solution and precipitation of material that is soluble in the developer.

The increase in the hydrophilicity of the polymer or a reduction in interfacial energy between the developer and the polymer components also results in higher chain/particle solvation and a corresponding reduction in particle generation. The impact of resist surface properties on particle formation can help to guide material design. For example lactones are incorporated in $193 \mathrm{~nm}$ resists to increase the polarity of very hydrophobic alicyclic polymers [14]. Based on the observations presented above, this would also be expected to assist in suppressing the particle generation. However, increased hydrophilicity may result polymer swelling and poor patterning due to the low solubility contrast. Therefore the optimal hydrophilicity of polymer components is, like most things in resist design, a trade-off. 


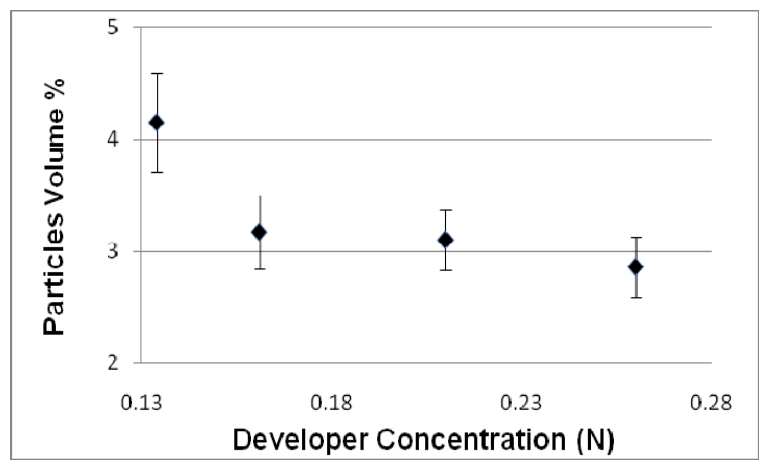

(a)

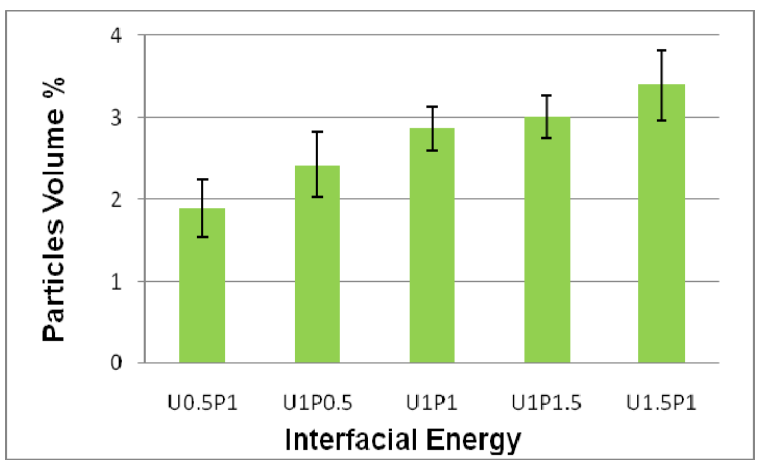

(b)

Figure 8. Particle volume $\%$ as a function of (a) developer concentration and (b) the interfacial energy of polymer components with the developer. $U$ and $P$ represent the unprotected and the protected parts of the polymer, where UmPn would mean that the interfacial energies of the unprotected and the protected parts are increased $\mathrm{m}$ and $\mathrm{n}$ times respectively.

\section{CONCLUSIONS}

A mesoscale model has been adapted to study particle generation during photoresist dissolution. New PEB and dissolution models have been incorporated that allow exploration of statistical excursions in the development path due to variance around the mean protection level. These excursions finally lead to line edge roughness and the formation of insoluble residues. Insoluble particles are generated during the photoresist dissolution when there is a connected pathway of soluble material that envelops a volume an insoluble material. An extensive parametric study was done to assess the extent of particle generation as a function of polymer formulation (degree of polymerization, protection level), exposure (dose, NILS), PEB (PAG/ Quencher loading, PEB time) and development (developer concentration, polymer hydrophilicity). Resist formulations and process conditions can be tailored to minimize insoluble particle generation. Overall, this study may provide a means to guide material design and optimize the process conditions for lower particle generation.

\section{ACKNOWLEDGEMENTS}

The authors wish to thank the Tokyo Electron Limited (TEL) for funding this work; Texas Advanced Computing Center (TACC) at The University of Texas at Austin for providing computing resources; Dr. Vishwanath H. Dalvi and Willson Research Group for advice and useful discussions.

\section{REFERENCES}

1. Schmid, G.M., Understanding molecular scale effects during photoresist processing. $\mathrm{PhD}$ thesis, The University of Texas at Austin, 2003.

2. Meiring, J.E., et al., "Using Mesoscale Simulation to Explore Photoresist Line Edge Roughness," Proceedings of SPIE 5753, 350-360 (2005).

3. Siddharth Chauhan, Mark Somervell, Steven Scheer, Chris A. Mack, Roger T. Bonnecaze and C. Grant Willson, "Polymer Dissolution Model: An Energy Adaptation of the Critical Ionization Theory," Proceedings of SPIE 7273, 727336/1-727336/11 (2009).

4. Flanagin, L.W., et al., "Probabilistic model for the mechanism of phenolic polymer dissolution," Proc. SPIE-Int. Soc. Opt. Eng., 1998. 3333 (Pt. 1, Advances in Resist Technology and Processing XV): p. 268-277.

5. Michael Carcasi, Mark Somervell, Steven Scheer, Siddharth Chauhan, Jeffery Strahan and C. G. Willson, "Comparison study of 248-nm/193-nm LWR using dynamic Monte Carlo molecular scale modeling," Proceedings of SPIE $7639(2010)$.

6. Stewart, M.D., Catalyst Diffusion in Positive-Tone Chemically Amplified Photoresists. PhD thesis, The University of Texas at Austin, 2003. 
7. Postnikov, S. V., Transport Properties of Photogenerated Acid and Silylating Agent in Polymer Films. PhD thesis, The University of Texas at Austin, 1999.

8. A. Reiser, J.P. Huang, X. He, T.F. Yeh, S. Jha, H.Y. Shih, M.S. Kim, Y.K. Han, K. Yan, "The molecular mechanism of novolak-diazonaphthoquinone resists," European Polymer Journal 38 (2002) 619-629.

9. Ozisik, M. N., Heat Conduction. 1993, 3rd edition, John Wiley \& Sons.

10. Schmid, G.M., et al., "Electrostatic effects during dissolution of positive tone photoresists," J. Vac. Sci. Technol., B, 2002. 20(6): p. 2913-2919.

11. Colburn, M.E., Step and flash imprint lithography: a low-pressure, room-temperature nanoimprint lithography. $\mathrm{PhD}$ thesis, The University of Texas at Austin, 2001.

12. Van Oss, C.J., R.J. Good, and M.K. Chaudhury, "Additive and nonadditive surface tension components and the interpretation of contact angles," Langmuir, 1988. 4(4): p. 884-91.

13. Israelachvili, J.N., Intermolecular and surface forces : with applications to colloidal and biological systems. 1985, London ; Orlando, [Fla]. Academic Press. xv, 296 p.

14. Hiroshi Ito, Hao D. Truong and Phil J. Brock, "Lactones in $193 \mathrm{~nm}$ resists: What do they do?," Proceedings of SPIE 6923, p.692318/1-692318/12 (2008). 